Cahiers Charlevoix

Études franco-ontariennes
Cahiers Charlevoix Études franco-ontariennes

or Crevenerix of

\title{
Là où il ne fallait pas de réponse. Réponse à " Désespoir de vieille fille " par Marie de Villers
}

\section{Fernand Dorais}

Volume 2, 1997

URI : https://id.erudit.org/iderudit/1039458ar

DOI : https://doi.org/10.7202/1039458ar

Aller au sommaire du numéro

\section{Éditeur(s)}

Société Charlevoix

Presses de l’Université d'Ottawa

ISSN

1203-4371 (imprimé)

2371-6878 (numérique)

Découvrir la revue

Citer cet article

Dorais, F. (1997). Là où il ne fallait pas de réponse. Réponse à « Désespoir de vieille fille » par Marie de Villers. Cahiers Charlevoix, 2, 351-387.

https://doi.org/10.7202/1039458ar
Résumé de l'article

Fernand Dorais, qui a présenté, dans un article précédent, le livre choc de Thérèse Tardif, Désespoir de vieille fille, considère maintenant Réponse à "Désespoir de vieille fille ». Cet ouvrage, que Simone Routier publia, six mois plus tard, sous le pseudonyme de Marie de Villers, est une réfutation vive, fragment par fragment, du livre de Tardif. Faute de pouvoir restituer tout le contexte socioculturel des années quarante, l'auteur étudie le malentendu profond qui séparait ces deux femmes et qui reposait sur des positions religieuses diamétralement opposées. Il montre surtout que, à la conception existentielle et marginale de la première, il ne fallait pas répondre par l'orthodoxie conceptuelle de la seconde qui dicte et condamne systématiquement, mais plutôt par la compréhension. 


\section{LÀ OÙ IL NE FALLAIT PAS DE RÉPONSE. RÉPONSE À «DÉSESPOIR DE VIEILLE FILLE» PAR MARIE DE VILLERS}

Fernand Dorais

Maison des jésuites, Saint-Jérôme

Cahiers Charlevoix 2, 1997, pp. 351-387. 


\section{SOMMAIRE}

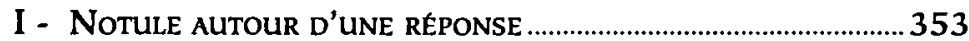

II - L'IRRÉDUCTIBILITÉ D'UNE OPPOSITION .............................................363

III - DeS DEUX PRÉSENCES AU MONDE ……………………….............. 379 


\section{LÀ OÙ IL NE FALLAIT PAS DE RÉPONSE. RÉPONSE À «DÉSESPOIR DE VIEILLE FILLE» PAR MARIE DE VILLERS}

Je demande à ceux qui aiment Dieu de prier pour vous.

Marie de Villers

\section{I - NoTUle AuTOUR D'UNE RÉPONSE}

Si «le 3 mars 1943», on «achev[ait] d'imprimer à l'imprimerie Saint-Joseph, à Montréal, pour les éditions de l'Arbre", un ouvrage intitulé Désespoir de vieille fille, signé Thérèse Tardif ${ }^{1}$, le 24 septembre 1943, aux Éditions Beauchemin, on «achevait d'imprimer" Réponse à "Désespoir de vieille fille»,

1 Thérèse Tardif $[=T T$, dans notre texte], Désespoir de vieille fille [désormais: $D V F$ ], Montréal, L'Arbre, 1943,124 p. On peut lire à la dernlère page numérotée (p. 124): «Ottawa-Hull 1937-1942». Les premières lignes du présent article ne font que répéter le début d'un texte qui a paru dans les Cahiers Charlevoix 1. Etudes francoontariennes, Sudbury, Prise de parole, 1995, pp. [361]-409: «La Mémoire oubllée en Ontario françals. Désespoir de vieille fille de Thérèse Tardif». 
réfutation indignée signée Marie de Villers ${ }^{2}$, alias Simone Routier. Pourquoi ce pseudonyme et d'où vient-il? Nulle réponse à ce jour. Par ailleurs, Villers, même ennobli du de obligé, consultation faite, ne réfère à rien de connu ou de célèbre, alors qu'à la date de la publication de son essai, madame Routier était loin d'être une inconnue dans les lettres québécoises ${ }^{3}$ et que le recours à un pseudonyme avait de fortes chances, comme mèche, d'être vite éventé ${ }^{4}$ Ici donc aussi, impossibilité d'élucider le recours en cause de la part de Simone Routier.

${ }^{2}$ Réponse d̀ "Désespoir de vieille fille [désormais: RDVF], Montréal, Éditions Beauchemin, 1943, 125 p. J'ignore ce qui a bien pu amener Suzanne Faguy à écrire Marie de Villiers dans le Dictionnaire des cuvres littéraires du Québec [désormais: DOLQ], tome 3: 1940-1959, sous la direction de Maurice Lemire, Montréal, Fides, 1982, pp. 276278 (compte rendu par Suzanne Faguy). Le Dictionnaire des auteurs de langue française en Amérique du Nord (Montréal, Fides, 1989, p. 1180) répétera cette erreur. On peut s'étonner d'une faute si gratuite.

${ }^{3}$ Simone Routier $[=S R$, dans notre texte], n'avait-elle pas mérité le prix David en 1929 (colauréate) pour son recueil de poèmes l'Immortel Adolescent (Québec, Éditions «le Soleil», 1928, 190 p.)!

${ }^{4}$ De trois comptes rendus qui ont paru peu après la publication de l'ouvrage, soit en janvier 1944 (Raymond Tanghe, "L'Éternel féminin", L'Action universitaire, vol. 10, n 5, p. 22), mars 1944 (GabrielM. Lussier, «L'Esprit des livres. Marie de Villers, Réponse d̀ «Désespoir de vieille fille», Revue dominicaine, vol. 50, pp. 190-192) et avril-juin 1944 (Hilaire [père], «Bibliographie. Marie de Villers, Réponse d "Désespoir de vieille fille», Revue de l'Université d'Ottawa, vol. 14, $\mathrm{n}^{\circ} 2$, pp. 255-256), aucun ne permet d'induire cette hypothèse. Autrement dit, personne, de facto, n'évente la mèche, mais l'un ou l'autre des critiques, qui étaient proches du monde de l'édition ainsi que de celui de l'auteur, devait bien savoir. Il est fort probable que, dans le milieu littéraire outaouais de 1943 , on a découvert assez rapidement qui se dissimulait sous le pseudonyme de Marie de Villers. Quoi qu'il en soit, dès l'année suivante, Simone Routier dévollait au grand public qu'elle était bien l'auteur de Réponse à "Désespoir de vieille fille», car elle ajoutait ce titre à la liste de ses ouvrages sur la page intitulée $« \mathrm{Du}$ même auteur" (p. [6] au début de la cinquième édition, revue et considérablement augmentée de son Adieu, Paris! Journal d'une évacuée canadienne, $10 \mathrm{mai}-31$ août 1940, Montréal, Éditions Beauchemin, 1944, 198[1] p. Elle prenait même le soin de préciser qu'elle avait publié ce volume «sous le pseudonyme de Marie de 
Sa Réponse, Simone Routier l'a rédigée en une seule petite semaine: "C'est pour celles-là surtout qui ont cru devoir prendre au pied de la lettre votre jonglerie de mots [celle de TT], que je me suis imposée [sic], - au seul détriment de mes contes aux enfants, une semaine durant ${ }^{5}-$, la récréation-corvée de cette réponse" (90-333) ${ }^{6}$.

Ces premières données soulèvent déjà au moins les quelques questions inévitables suivantes.

1. D'abord, pour lire l'ouvrage de $S R$, et surtout pour le comprendre vraiment, ne faudrait-il pas avoir lu TT? La réponse de $S R$ est claire: «Un des reproches qu'on me fera est celui de pousser mes lecteurs à l'emprunt, sinon à l'achat, de votre Désespoir, quoique, à la rigueur, cette Réponse puisse être lue indépendamment" (90-330). Cette affirmation est insoutenable. Quiconque feuillette RDVF se voit vite renvoyé à l'ouvrage de $T T$, sous peine de lui voir échapper le sens de la plupart des fragments. Un seul petit exemple, entre dix autres possibles, convainc. Le fragment 195 (p. 60) se lit: «Si, si!». Or voilà qui demeure tout à fait inintelligible, si l'on ne consulte pas l'ouvrage de TT, et même après l'avoir consulté! En effet, $T$ T avait écrit: «Les... n'ont rien à envier aux Pasquier». À quoi $S R$ rétorque ce qu'on sait. Retenant

Villers». En 1981, six ans avant sa mort, elle inscrivait encore Réponse d̀ «Désespoir de vieille fille» avec la même précision sur la liste de ses ouvrages à la page 6 de ce qui devait être son dernier ouvrage, le Choix de Simone Routier dans l'ceuvre de Simone Routier, Charlesbourg, les Presses Laurentiennes, "Le Choix de...», 1981, 79 p.

${ }^{5} R D V F$, p. 124: «10-17 mai 1943». Donc deux mois après la publication de l'ouvrage de Thérèse Tardif, la Réponse est prête.

${ }^{6}$ Le premier nombre renvole à la page de l'œuvre (ici: 90); le second, au numéro du fragment cité (ici: fragment 333). Cette dernière numérotation est de nous; elle n'existe donc que dans les exemplaires que nous avons utilisés. 
précisément l'exemple en question, un critique commente: «Le genre admet beaucoup de fantaisie, et quand Marie de Villers répond par ‘Si, sil! à l'absurde ‘Les... n'ont rien à envier aux Pasquier, j'en suis enchanté. Et j'applaudis encore $[\ldots]^{7}$ ॥l faut toutefois concéder que, dans moult cas, le lecteur n'est diable plus éclairé sur le sens des réfutations de $S R$; encore faut-il comparer la source et la réponse, au cas où quelque lumière naîtrait pour éclairer les fragments, nombreux, qui ne livrent pas encore leur secret de signification.

Voilà qui amorce un autre problème, celui de la comparaison entre les deux ouvrages. TT avait publié un "essai» composé de 466 fragments, dûment séparés les uns des autres ou tout à fait autonomes. $S R$ entend reprendre chacun de ces fragments et lui donner sa réplique, soit sa "réponse», un écho, une correction, une rectification, voire le plus souvent une condamnation. C'est ainsi que les deux ouvrages se «correspondent»: l'un propose, l'autre dispose, tout comme Dieu ou, encore pis, comme l'Inquisition. Pour illustrer ce phénomène, prenons par exemple au hasard les pages 58-59. La première (58) compte huit fragments dans l'un et l'autre ouvrage, et ces fragments sont numérotés: 180-187. Il en va de même pour la page 59 , qui compte dans les deux livres six fragments, affectés des mêmes numéros: 188-193. RDVF se présente donc comme une reprise et une relancée du texte de TT. Cette correspondance n'est pas absolue. Ici et là, à l'occasion, le nombre de fragments pour telle page donnée diffère d'un auteur à l'autre; et c'est le lecteur qui

${ }^{7}$ Gabriel M. Lussier, «L'Esprit des livres. Marie de Villers, Réponse à "Désespoir de vieille fille", Revue dominicaine, vol. 50, mars 1944, p. 191. 
paie la note de cette dissymétrie, cherchant bien, sans le trouver souvent, à quel texte renvoie $S R$. Mais, ce sont là inévitables accidents de parcours.

2. La véritable question que pose $R D V F$ reste la suivante: quelle mouche a bien pu piquer madame Routier pour que, Désespoir de vieille fille à peine publié, elle mette tout de côté et en rédige, à toute allure, une réfutation plutôt dure en moins d'une semaine? Qu'est-ce qui, dans le texte de TT, la fait vomir? Terme trop fort?... C'est $S R$ elle-même qui l'emploiera, ainsi que "nous l'allons montrer tout à l'heure ${ }^{8}$ ».

Notre question ne pourrait recevoir de réponse valable que si la biographie des deux femmes de lettres existait, biographie scientifique qui restituerait tout le contexte socioculturel dans lequel $T T$ et $S R$ ont été élevées et ont vécu. Leur psychologie sous toutes ses formes y serait examinée, avec le recours aux journaux et carnets intimes, cahiers de notes, mémoires et correspondance, qu'elles ont pu laisser. Alors, et alors seulement, pourrions-nous sérieusement traiter le problème qui est au fond de RDVF et de notre questionnement. Faute de pareils instruments, nous en restons presque réduits à des balbutiements, par essais et erreurs. Toutefois, comme nous le prétendons à la lecture et aux relectures des deux ouvrages en question ici, quelque chose de très profond oppose les deux écrivains, et c'est leur attitude respective à l'endroit de la religion catholique alors pratiquée à l'unanimité dans la "sainte» province de Québec, ce sur quoi se concentre le présent article.

8 «La ralson du plus fort est toujours la metlleure:

Nous l'allons montrer tout à l'heure.»

La Fontaine, "Le Loup et l'Agneau», Fables, livre premier, fable X. 
Cette réaction, qui nous intéresse, de $S R$ devant le livre de $T T$ et son auteur peut être décrite comme suit. Plus d'un passage enregistre le dégoût que $D V F$ inspire à $S R$. Là où $T T$ avait écrit: "Il n'y a que possibilité de haine dans le monde. Tout ce qui est amour est voulu et artificiel» $(68-228), S R$ répond: "Que le prêtre a de vertu, grand Dieu! qui doit assister à pareils déshabillages et dégobillages (pardon lecteur) à cœur de confessions». Les grands moralistes français du dix-septième siècle nous avaient pourtant habitués à pareille vision janséniste de la méchanceté dans l'homme: «La raison du plus fort...», ce n'est pas le hasard ou le caprice qui nous fit plus haut citer ces vers, et nous tairons ici, par crainte d'être prolixe, La Rochefoucauld. Enchaînons. Et un peu plus loin, à propos de cette remarque de TT: «Il faut passer à travers le monde les narines appuyées sur les pouces" (82-293) ${ }^{9}$, madame Routier commente: «Et avec de l'eau de Cologne dans le mouchoir pour lire vos élucubrations» $(82-293)$. «Après certaines de vos réflexions on a envie de courir se laver les mains» (69-236). "Publier pareil livre à pareille époque de pareilles viriles souffrances!» (61204; allusion évidemment à la guerre de 1939-1945). Et pour clore péremptoirement ce petit répertoire: «Et quand on pense que la mort pourrait vous surprendre au milieu de pareille page» (63-214). Le lecteur s'empresse d'aller lire la "page» en question: "Il faut croire et ne rien attendre. Espérer. Je ne sais pas offrir; mais que l'on exige, tant et tant et jusqu'à

9 Pour ouvrir, ut sic dicam, un peu notre texte, portant sur l'essai, rappelons le mot de Chamfort à propos du sujet même traité ici par TT: «M. de Launay, homme très doux, mais qui avait une grande connaissance de la société, disait qu'il faudrait avaler un crapaud tous les matins, pour ne trouver plus rien de dégoûtant le reste de la journée, quand on devait la passer dans le monde.» 
la fin! Je me traînerai, bien sûr, heureuse sous mon visage de misère, vers mes amis que j'aime" (63214). Le lecteur tombe de haut! Conséquence et conclusion: TT devrait et va éprouver le remords d'avoir jamais écrit ce livre: «Le remords, c'est ce qui s'emparera peut-être de vous lorsque, mère de famille, vous verrez votre première œuvre dans les mains de vos enfants» (97-377). Et voilà pour le conseil charitable du «juste» accusant et condamnant: «que ne vous êtes-vous pas abstenue! ${ }^{10}$ ».

Non sans quelque arbitraire ici dans le développement logique du texte, récapitulons.

A. Pour ce qui est du livre lui-même et en lui-même.

1. Quelle est la forme de son livre? «Ceci est une réponse (avec pagination correspondante) à ces pages où il est beaucoup parlé du péché par une âme en détresse» (p. 9).

2. Pourquoi $S R$ a-t-elle entrepris de l'écrire? Pour prévenir et enrayer le scandale que pourrait produire DVF: «Un péché vendu - mettons celui du scandale d'un livre - encourt plus de responsabilité que le péché gratuitement accordé à cause de la complicité qu'il réclame» (96-372). La lecture de TT pourrait troubler certaines âmes non prêtes à le recevoir ou

\footnotetext{
${ }^{10}$ Ici, s'imposerait, selon l'esprit croyant de l'époque, l'épisode de la femme adultère: "Alors, se redressant, Jésus lui dit: ‘Femme, où sontils? Personne ne t'a condamnée?, Elle dit: (Personne, Seigneur.) Alors Jésus lui dit: «Moi non plus, je ne te condamne pas. Va, désormais ne pèche plus.» (Évangile selon saint Jean, chap. 8, versets 10-11.) Et plus loin dans le même Évangile (chap. 9, versets 1-3), un autre épisode viendrait confirmer cette "non-condamnation" chez le vrai juste; «En passant, il vit un homme aveugle de naissance. Ses disciples lui demandèrent: "Rabbi, qui a péché, lui ou ses parents, pour qu'il soit né aveugle?» Jésus répondit: «Ni lui ni ses parents n'ont péché, mais c'est afin que soient manifestées en lui les œuvres de Dieu.»
} 
mal prévenues devant ses «jongleries»: «C'est pour celles-là surtout qui ont cru devoir prendre au pied de la lettre votre jonglerie de mots..." (90-333, déjà cité plus haut).

3. $S R$, en jetant ainsi sur la place publique ses réactions hostiles à $D V F$, ne court-elle pas le risque de trop attirer l'attention sur cet essai regrettable, plutôt que d'en éloigner les curieux ou mieux les curieuses («celles-là») par trop innocentes? «Un des reproches qu'on me fera est celui de pousser mes lecteurs à l'emprunt, sinon à l'achat, de votre Désespoir...» (90-330).

4. Au fond, le scandale est-il donc si grand? Oui et non; mais, toutefois: «Ce livre est mauvais, mais pas troublant» (24-55). Et, par ailleurs, $S R$ fait sienne la critique que Guy Sylvestre adresse à DVF: «Construction de la raison pure, ce livre laisse l'âme incomplètement satisfaite, car il ne fait pas suffisamment appel à notre sensibilité» (37-112), - lit-on pareil texte qu'on ne peut qu'avouer son impuissance à en comprendre quoi que ce soit: «Construction de raison pure... pas appel à notre sensibilité»!

5. Il y a encore plus, et plus obscur pour le lecteur actuel qui s'interroge sur ce qui s'est passé dans les coulisses au moment où $T T$ rédigeait son Désespoir. "J'allais reconnaître à votre ouvrage le mérite de n'être insupportable que parce que d'une vérité totale, sinon d'une vérité d'art telle qu'on l'attend de l'écrivain aussi bien que de tout artiste. J'ai eu la déception d'apprendre qu'il n'était qu'une gambade de "pitre» adroit (celui de la page 26$)^{11}$, une «auto-

\footnotetext{
"Voici le texte de TT auquel $S R$ fait allusion: «La vie est une heureuse comédie. Et celui qui refuse d'y particlper est un pitre. Je suis un pltre» (DVF 26-68).
} 
biographie chiquée" (même avec initiales et noms d'amis personnels) de bachelière en mal de publicité» (85-320). Le lecteur se voit soudainement confronté à une imposture. Des confidences, indiscrétions, bavardages (des amis communs: ?...) ont révélé à $S R$ qu'il y a beaucoup de pose dans ce petit essai sur les tristes et pauvres souffrances d'une vieille fille abandonnée du seul amant qui semble avoir compté dans sa vie et qui s'est sauvé pour peut-être (vraisemblablement!) la laisser seule avec un "enfant" conçu dans le péché, au vu et au su de quelques gens au moins! Madame Tardif a tout noirci, inventé des noms, surenchéri, etc., pour se rendre intéressante, attirer l'attention, voire (une fois partie) percer dans le monde littéraire.

6. Tout aboutit à de la littérature: «M'est avis que, là encore, il y a bien quelque littérature» (95-365). Il fallait bien trouver le trouble et la honte psychologique qui expliquait la publication d'un tel essai nauséabond. «La vie» de l'auteur en répond. On détient la clé de l'œuvre, tout comme naguère encore, à la même époque, un prêtre quittait-il la soutane qu'on s'interrogeait sur la raison vraie et dernière de cette trahison: «Cherchez la femme, disait-on, et vous aurez le motif honteux de cette défection ${ }^{12}{ }^{\prime}$. L'œuvre n'avait plus tellement besoin d'autres grilles de lecture et d'interpétation. On venait d'en découvrir le vice profond.

12 Un chanoine, célèbre prédicateur de retraites de décision (ou de choix de carrière) à l'époque, tâchait d'enrôler le plus de collégiens possible dans le sacerdoce, convaincu que tout étudiant avait la vocation à la prêtrise et que, si l'on ne répondait pas à cet appel de Dieu, c'était par manque de générosité. Et au fond l'on n'ignorait point la cause de ce refus. La femme (et la peur du sacrifice qu'imposait la chasteté), tel était le principal ennemi de l'appel de Dieu dans l'âme de nos petits chrétiens, déjà tout atteints de l'esprit du siècle, soit le laxisme américain. 
Ainsi d'ailleurs, et trop souvent, la critique catholique officielle procédait-elle pour "comprendre" et juger une œuvre. On allait fouiller les vidanges de la vie de Baudelaire, Lautréamont, Verlaine, Rimbaud, puis on disait: "Vous voyez; que peut-il sortir de bon de pareilles poubelles?» (Tout comme on lit dans le Nouveau Testament: "Que peut-il sortir de bon de Nazareth?») Au nom de la vie intime de l'auteur, l'œuvre se trouvait irrémédiablement atteinte et ne pouvait être que dangereusement malsaine et pernicieuse, voilà les deux mots clefs sortis du sac: «Comme vous aimez surveiller les allées et venues de cette sorte de littérature, de stercoraires. Qui se ressemble...» (70-241; sans doute ici le jugement le plus sévère et injuste que prononcera $S R$ au sujet de $D V F$ ).

7. Cette révélation faite, madame Routier peut écrire dès le début de sa réfutation: "Je les ai lues ces pages, avec avidité. Je me suis rassasiée, sans joie, de leurs péchés, et me suis dit: comme cette femme a mal appris de la vie» (p. 9, en exergue! ${ }^{13}$ ). Mais de quels péchés madame Routier peut-elle bien parler, se demande-t-on après avoir lu les deux ouvrages? Sera amorcée plus bas une piste d'explicitation.

B. Pour l'instant, enfin, question de déblayer quelque peu et de compléter les entours de l'œuvre à l'étude, $R D V F$, il serait bon de se demander de quel œil madame Routier voit la pécheresse TT?

Et l'auteur et son œuvre (on vient de le dire) ne peuvent s'attirer, se mériter, que mépris ${ }^{14}$ profond,

\footnotetext{
${ }^{13}$ Et madame Routier, résumant l'expérience que lui a fait faire DVF, conclura: «On quitte ce livre avec le même regret qu'on s'arrache une vieille gale» (124-466).

${ }^{14}$ Là où TT avait écrit: "Nous méprisons les autres parce que nous sommes nous-mêmes remplis de péchés» (98-385), madame Routier
} 
non sans hargne, de la part de $S R$. Est-il vraiment une page de RDVF, une seule qui, d'une façon ou d'une autre, ne comporte pas d'insulte à l'endroit de TT et de son essai? Se trahit sans cesse, affleure partout, quelque chose comme une vaste lame de fond de répugnance, de répulsion, voire de haine viscérale, en ce qui concerne l'attitude fondamentale de TT à l'égard de Dieu et de la vie (et ses grands thèmes indécidables, tels l'amour, l'amitié, le sens ultime de l'existence, la souffrance, etc.).

\section{II - L'IRRÉdUCTIBIUITÉ d'UNE OPPOSITION}

Madame Routier va se situer en face de Thérèse Tardif, avouant le «lieu» d'où elle prend la parole: «...mais je n'ai jamais suivi le moindre petit cours de littérature, moi, ni dans ma ville, ni ailleurs et je ne suis même pas bachelière. Il vous est même permis de rétorquer ici que ça se voit..." (96-368). Toutefois, $S R$ n'en conseillera pas moins à son interlocutrice: «Recourez à votre petit catéchisme et laissez Saint [sic] Thomas tranquille» (92-341). Et SR d'ajouter aussitôt: «Mettre le feu à la pédante bibliothèque de votre cerveau, de vos réminiscences» (92-344), et «...ouvrez l'Imitation...» (56-172), sans négliger de revenir au simple bon sens des choses, des simples gens, de la vie qui «est là simple et tranquille». Ultime conseil, souvent répété d'ailleurs, de madame Routier à la Tardif ${ }^{15}$ : «dé-dramatiser» son existence,

répond: «Ce n'est pas autrement que j'ai pu vous mépriser à travers tant de ces pages» (98-385).

15 «Pardon, lecteur, je parlais à Tardif» (50-160, et :«Tardif joue son grand air...» (24-51). Parfois c'est sur ce ton cavalier que $S R$ s'adresse à ladite donzelle Tardif. Ce ton de petite supériorité et de leçon de choses, on ne l'a que trop connu au Québec, au temps de la dictature des clercs qui détenaient tout le bon sens possible et impossible 
écouter le sens commun et pratiquer la simplicité comme le grand air et la cuisine saine, rééquilibrer sa raison, son psychisme, et s'abandonner, pleine de confiance, au devenir et à la bonté du Père, soit, en un mot, émerger à la santé et se convertir à la réalité quotidienne vécue ${ }^{16}$ : «Fièvre exacerbée. Un midi brûlant d'août. Pourquoi ne pas aller vous asseoir, en maillot de bain, dans le plus proche ruisseau, ou dans votre baignoire pour écrire. Il y a déjà un petit moment que j'ai envie de vous parler eau froide" (49-151). Et voici un autre exemple éloquent de l'appel au bon sens, au sens commun, comme moyen de récupération au service de la bonne cause: «Que n'épousez-vous un homme d'affaires qui vous donne beaucoup d'enfants? Je tiens la recette pour bonne» (34-90). Mais la demoiselle Tardif n'est-elle pas irrémédiablement rongée par la "rage de provoquer» (35-93), qui la travaille et la force à jouer à la femme de lettres: «... n'ayant pu se faire chatte s'est faite femme-de-lettres» (63-217) ${ }^{17}$ ? Madame Routier a la recette de la cure qu'il faut à $T T:$ «... et secouez un peu... votre vermine et vos phrases de rtéléphones où l'on poses " (95-365).

Entre les deux femmes ne cessent de s'interposer

nécessaire et suffisant pour bien vivre et accomplir son salut! Des êtres de jugement, disait-on, alors que tous les autres, évidemment, manquaient de jugement. Ce sont des faits de culture, voire de civilisation comme ça qu'il faut rappeler dans la présente querelle: seuls, au fond, ils peuvent en reconstituer la toile de fond et en éclairer tout le contexte global, pour le restituer à sa juste et vraie valeur.

${ }^{16}$ Au principe de réalité, diraient peut-être les freudiens.

${ }^{17}$ Ce fragment est cité dans son entièreté, et répond au texte suivant de TT: «Nous avons besoin de l'humus des âmes pour recréer les âmes, de l'humus des corps pour recréer les corps» (63-217, quoi que cela puisse vouloir dire...). Quant à la forme de cette réponse, voir le fragment où $S R$ cite Victor Hugo: «La fidélité ne pouvant se faire homme s'est faite chien" (23-48). 
les malentendus les plus complets, loufoques parfois et parfois pénibles à force d'incompréhension de la part de $S R$, quand ce n'est pas de mauvaise foi ou de mauvaise volonté. Semble exister telle chose que la cécité volontaire, quand quelqu'un ne veut pas voir... Quelques exemples seulement pour voir ce dont il s'agit:

- TT: «La chasteté est la dignité du cœur dans l'amour" (107-403).

$S R$ : «La chasteté est la maîtrise de la sensualité» (ibid.).

- TT: «Je n'ai pas assez aimé mes amis. Je voudrais les avoir importunés de mon amour" (108408).

$S R$ : «On ne devrait s'autoriser à importuner son prochain que lorsqu'il s'agit de son intérêt et de son bonheur à lui. Et qui peut s'arroger le droit de juger pour un autre de son bonheur» (ibid.)?

- TT: "Aidez-nous à comprendre le péché des autres, à nous libérer du nôtre» $(121-449)^{18}$.

$S R$ : «Ne louche pas sur les poutres des autres, buvant ton «coke), pense à ta paille» (ibid.).

- TT: «Qu'importe le déluge.

Ce que j'aime, ce en quoi j'ai foi, c'est la religion qui nous a donné le Christ de Rimbaud mourant, le Christ fort de Claudel, le Christ des fuites de Mauriac, le Christ baigné de blasphèmes par Renan, le Christ adoré par son petit-fils Ernest Psichari» (121- 452).

\footnotetext{
${ }^{18}$ On pensera ici au mot de Tchékhov, cité de mémoire: «Tout comprendre c'est tout pardonner. Et il serait étrange alors de ne pardonner pas.» La réponse de $S R$ n'est certainement pas ce qu'elle a répliqué de mieux!
} 
(Il faudra sans faute revenir sur ce texte très fort, considérant surtout l'époque à laquelle il fut écrit.)

$S R$ : «Religion apprêtée à toutes les sauces, piquantes ou fades, des âmes en mal de pédanterie" (ibid.; il est difficile d'imaginer réponse tout simplement si «in-compréhensive», pour ne pas dire plus bête!).

Remarquable, dans les fragments cités ${ }^{19}$, la moralisation ou le ton prêchi-prêcha de la "répondante ${ }^{20}$ ", là où précisément il ne fallait pas de réponse, mais de la compréhension, qui met à l'écoute de l'Autre dans une attention et une attente ardentes d'où sourdent bientôt les vraies questions que posent nécessairement la recherche et la quête de l'Autre... encore (ce qui pour moi s'appelle l'authentique herméneutique: une maïeutique qui fait accoucher le texte de ses intentionnalités les plus profondes, n'est-ce pas là, au fond, le fait et la méthode de Paul Ricœur ${ }^{21}$ ?). On a l'impression de lire la Somme de saint Thomas dont les articles commencent invariablement avec la formule pour ainsi dire judiciaire: "Respondeo dicendum quod...» (i.e. je réponds qu'il faut dire que...). On a tout de suite toutes les réponses à toutes les objections possibles. Heureux homme qui possédait ainsi toute la vérité et avec elle

\footnotetext{
${ }^{19}$ On verra par le reste du texte que ces exemples n'ont pas été choisis au hasard.

${ }^{20}$ Autre exemple de cette leçon d'orthodoxie, qui répète son petit catéchisme et donne la réponse rectification attendue:

- TT: «L'amour est une ruse de la chair» (21-38).

$S R$ : «L'amour est l'appât que le Créateur a bien voulu ajouter au devoir du (Crolssez et multipllez).» (ibid.).

- TT: «Aimer, c'est créer la vie.» (p. 101 en exergue au chapitre IX).

$S R$ : «Aimer c'est permettre à Dieu de créer une ame." (ibid.).

${ }^{21}$ Voir là-dessus ce que pourrait être une pareille pratique de la quête du sens: George Steiner, Réelles Présences. Les Arts du sens, Paris, Gallimard, 1989, $280 \mathrm{p}$.
} 
s'identifiait, pour qu'elle puisse parler par sa bouche, attitude trop courante dans la vie et la pensée de l'Église catholique qui, identifiée à Dieu, peut à pied levé réfuter tous les adversaires de tous les pays, de tous les temps!

Puisque viennent d'être cités à la barre de la vérité et de l'histoire des fragments sur les thèmes de l'amitié et de l'amour, il ferait bon de compléter ce paysage par d'autres extraits, avant d'aborder le dernier et le plus important sujet de ces deux ouvrages: la religion, l'expérience de Dieu dans la vie de deux croyantes cultivées au début des années quarante.

Les principaux textes relatifs aux thèmes de l'affectivité se trouvent dans la section $I X$, qui porte précisément en exergue un apophtegme déjà cité: "Aimer, c'est permettre à Dieu de créer une âme» (p.101; position des plus orthodoxes... là où TT avait écrit: "Aimer, c'est créer la vie», conception autrement dynamique).

D'abord, une indication anthropologique chez $S R$ : "Quant à l'homme, en lui-même, il n'est toujours qu'un cul de bouteille qui ne vaut que par le soleil qui le traverse» (56-173). On veut bien ignorer ce qu'un pauvre freudien (s'il en existe encore!) ferait de ce «cul de bouteille», mais le moins qu'on puisse souligner, c'est la dureté, pour ne pas dire vraiment ici la grossièreté, ou mieux la vulgarité, et la portée, de cette vision de l'être humain. Il faut reconnaître toutefois une portée positive à la remarque de $S R$. L'homme est et n'est là que pour être traversé par la lumière de la grâce. Sa "corporalité» (ou "corporéité») fait obstacle à la possession de son être par Dieu, ou le Divin. Le tout de sa vie sera dès lors de travailler, de collaborer à l'effort de la "venue de Dieu» en lui 
en cessant d'y faire obstacle par le mal, le péché dont il est seul auteur. Il doit se rendre transparent à Dieu. Cette conception de la spiritualité était courante à l'époque, et s'appuyait sur des textes de mystiques peinant à définir ce que pouvait bien être l'expérience de Dieu. Il n'en reste pas moins que le texte de $S R$ retient quelque chose comme de méprisant et provocant. Et la formule que le lecteur conserve c'est: l'homme est "un cul...». Conception janséniste sous-jacente humiliant la nature humaine? Cette suggestion serait difficile à maintenir. Il faudrait pour cela prouver, établir, que telle était la pensée de l'auteur dans le reste de son œuvre, voire dans sa vie, et que, en cela, elle ne faisait que s'inspirer de la "religion» du temps et la refléter. Jusqu'à preuve du contraire, la publication, par exemple, de notes ou journaux intimes, rien ne semble indiquer qu'il en fut ainsi. L'explication restera à trouver ailleurs, ce qui sera tenté dans le traitement de la religion chez $S R$.

L'amour de Dieu demeure le critère, la norme, la mesure de l'amour humain ${ }^{22}$ : «Si l'amour de Dieu était le noyau solide au centre de votre âme, esprit et corps s'ajusteraient sans brisure» (44-123). L'amour humain, ainsi guidé, s'irradie dans la conduite humaine: «Dieu a voulu, écrivait Lacordaire, qu'aucun bien ne se fît à l'homme qu'en l'aimant, et que l'insensibilité fût à jamais incapable, soit de lui donner de la lumière, soit de lui inspirer de la vertu" (44-124) ${ }^{23}$.

\footnotetext{
${ }^{22}$ Le mot est pris ici au sens où les Grecs disaient que l'Homme est la mesure de toute chose.

${ }^{23} \AA$ n'oublier pas que le même Lacordaire avait déjà écrit: «Que le prêtre soit avare, orgueilleux, pharisien: tant que le signe de la chasteté restera sur son front, Dieu et les hommes lui pardonneront beaucoup».
} 
Par ailleurs, tourmente toujours la chair, qui rend l'homme semblable à la bête et rabaisse l'amour aux gestes dégradants de la possession passionnelle: "Certains êtres aiment renverser les rôles et se faire esclaves de la chair parce que la dignité leur répugne et qu'ils ont les reins serviles» (45-125). "Le cul...» revient ici: «L'amour dans sa fureur est chose si laide (remarque Léonard de Vinci qui sur toute chose portait un regard d'esthète), que la race humaine s'éteindrait, - la natura si perderebbe, - si ceux qui le font se voyaient» (45-130). La vision et la conception du «rapt d'amour» dégrade l'être humain, qui, s'observant le faire, éprouverait un dégoût insurmontable et resterait incapable de sexe... On s'interroge s'il ne s'agit pas ici d'un lapsus, par exagération, chez $S R$; ou, pour le mettre en d'autres termes, un geste, un mot de durcissement dû à l'impatience, et encore bien mettre sa pensée en évidence en la durcissant à cette fin (le reste de l'ouvrage autorise pareille mise en perspective du texte cité). Mais la citation serait digne de tel passage de saint Augustin, qui, dans l'une de ses homélies les plus regrettables, soutenait devant Hippone réunie pour la messe dominicale: "Quand les parents (chrétiens) s'unissent dans le lit conjugal pour donner la vie, la chambre alors devient un bordel et la femme une meretrix (crûment une putain dans une traduction fidèle ${ }^{24}{ }^{2}$ " Convient donc de distinguer désir et amour, et de réhabiliter ainsi ce dernier: "Le désir use, mais l'amour embellit, aussi longtemps qu'il demeure l'amour. Qui ne sait cela?» Tantôt madame $S R$ ne semblait pas le savoir, si bien qu'un lecteur quelconque aurait pu lui poser à pied levé, comme ça, si elle savait comment se font des

\footnotetext{
${ }^{24}$ Encore faudrait-1l donner la référence exacte à ce texte plus qu'étonnant, ce qui échappe icl.
} 
enfants..., ce qui eût été certes le comble du ridicule... Amour, chasteté, sexe, mal: tout le cortège habituel retrouvé, avec les réponses orthodoxes récitées comme dans le petit catéchisme, stéréotypes où s'englue et se perd le texte de madame Routier. À preuve quelques remarques tirées de RDVF: «L'amour est l'appât que le Créateur a bien voulu ajouter au devoir du «Croissez et multipliez〉 " (21-38); il y eut donc le devoir de faire et d'avoir des enfants, vint s'y ajouter du désir, quelque appétit pour faire avaler cette obligation de la fécondité naturelle aux espèces animales; discours trop souvent entendu à l'époque que celui de ce petit prix de consolation pour compenser les immenses sacrifices de la fondation d'un foyer et d'une famille; vision qui nie toute valeur et toute qualité intrinsèques de l'acte de plénitude et de création qu'est le don physique du sperme. Aussi, madame Routier peut-elle ajouter: «La chasteté est la maîtrise de la sensualité" (107-403; là où $T T$ avait écrit: "La chasteté est la dignité du cœur dans l'amour» [107-403]). Aussi encore ce reproche si injuste à DVF: «D'ailleurs les péchés de l'amour par vous décrits ne sont même plus des tentations. Votre regard acidulé les décape de toute sensualité, de tout attrait, parce que de toute pudeur" (72-260). TT ne connaît que trop bien l'amour apache de l'amant, dirons-nous, occasionnel ${ }^{25}$. Ou alors madame Tardif joue à l'agace, l'aguicheuse: «Vos agaceries, reculs et marchandages à l'amour sont plus odieux que l'abandon trop généreux de maints catastrophiques consentements» (95-363).

\footnotetext{
${ }^{25}$ Voir texte de TT: «Celui qui viendra, si tard, il faudra qu'il m'aime nue, hagarde, échevelée» (63-213). Ce à quoi SR répond: "S'il a des goûts d'apache et n'a jamais fréquenté que des filles, ça pourra aller" (63-213).
} 
Bref, resituons le débat dans la lumière et la perspective des deux essais. Et $T T$ et $S R$ ont connu un grand amour malheureux. De ce qui paraît être l'amant de la première, il est dit qu'il ne l'aimait pas, qu'il butinait ailleurs, alors qu'elle lui avait voué son cœur et son corps; il ne la voulait que comme une amie à qui il pût confier tout de lui-même, et elle consentait à ce jeu de confidente que chaque nouvel aveu atteignait et blessait profondément dans son désir d'être l'élue, (surtout s'il y a eu enfant...). C'est du fond de l'abîme que crie au Seigneur, à la révolte intime et secrète, la femme stigmatisée par l'humiliation de n'avoir pas été choisie, d'avoir été délaissée, pour jouer un nouveau rôle chez l'amant d'une nuit: être celle dont Dieu se sert pour le ramener à Lui et l'instituer en sainteté. Voilà ce que confesse le poème inaugural, "Augustin renvoyant la femme» (p. 11): "Dieu concède la gloire aux plus faibles de ses saints. Ceux qui servirent à leur sanctification sont perdus dans l'oublì (p. 14). La femme ne serait plus là que l'instrument privilégié dans le retour à Dieu et le cheminement dans les voies de la sainteté de l'être hier encore passionnément désiré. Elle s'efface, ayant conduit comme par la main l'homme à son Maître et Père, et on l'oublie bien vite... N'est-ce pas là d'ailleurs, et à cette époque-là déjà, le rôle et la fonction de la femme se sacrifiant pour que, de l'homme, le saint émerge? N'est-ce, ou n'était-ce pas là la conception de la femme chez un Claudel, par exemple $^{26}$ ? DVF est le chant douloureux de la femme,

${ }^{26}$ On trouvera clairement exprimée et chantée cette vocation de la femme dans la cinquième et dernière des Cinq Grandes Odes: la Maison fermée. Le titre à lui seul résume bien la mission féminine de l'épouse, qui est de protéger le poète contre lui-même dans d'épuisantes quêtes d'amour impossible, de chairs toujours insatisfaites et humiliantes, de restlessness. Elle ferme la porte de la maison, du foyer, 
tour à tour ou à la fois révoltée et résignée, pour laisser toute la place au Maître des cœurs et des âmes. Mais elle a dû passer par le mal, le péché, et comme Job s'en prendre amèrement à Dieu qui oblige à de pareils sacrifices. Encore une fois, c'est du fond de l'abîme qu'elle a pris la parole; et c'est âpre. Il s'agit d'un cheminement spirituel parcouru dans un désert de sécheresse humaine et spirituelle, au cours duquel l'auteur sut rester fidèle aux données humaines de son expérience, à l'appel au mal pour que l'homme, et la femme, puissent s'accomplir.

Pour $S R$, c'est à un tout autre type d'expérience que nous avons affaire. Remarquons tout de go que madame Routier a trop bien connu l'amour pour le mépriser, ou mieux pour mépriser son Jean:

Il me reste de toi le souvenir de toute la joie qu'un être humain, radieux de force, de coeur et de beauté, peut offrir à la femme de sa dilection. Toi seul, après Dieu, savais avec quelle âpre patience nous nous étions attendus, à quelles abruptes hauteurs nos routes s'étaient confondues, de quel regard nous nous étions compris et vibrants de quelle témérité nous défoncions l'avenir pour toute la vie et pour tout le passé à s'apprendre. C'est à la lueur d'une haie de cierges en escorte dans la maison des vivants, que j'évoque à présent le trop parfait sourire, à jamais souriant, de ton trop cher visage (108-409; c'est peut-être ce que $S R$ a écrit de mieux dans sa Réponse).

de la famille, aux chimériques sollicitations du "dehors», de l'extérieur, de l'infini, des horizons et des débauches à la Rimbaud-Verlaine. La femme confère son statut social à l'homme: respectabilité, et d'abord dans la communauté où il habite; fécondité attendue, saine et normale, etc., alors que le démon de midi pousserait cet inguérissable don Juan entré dans la quarantaine vers des aventures sans fin comme sans lendemain. La femme "clôt» l'homme, elle l'encercle, en le protégeant contre lui-même, avec d'ailleurs la complicité du mâle quí ne demande pas mieux que d'être ainsi pris en charge, volre parfois castré, assagi, rangé, pour produire son œuvre et devenir utile à la société. 
Mais madame Routier se méfie des délires de l'amour non orthodoxe. Il faudrait reprendre ici les nombreuses citations sur l'amour rapportées plus haut et en faire une exégèse serrée qui prouverait notre thèse. Voilà qui serait trop long et fastidieux pour le texte que nous entendons présenter ici, et qui n'est qu'une amorce, une première ébauche d'un travail à poursuivre et à approfondir. Un fait cependant demeure révélateur, c'est l'assèchement progressif par la rationalisation et le moralisme qu'on relève dans l'œuvre poétique de Simone Routier. À cet effet on peut prendre à témoin les trois femmes auteurs qui en présentent les recueils (chacune indépendamment des deux autres) dans le Dictionnaire des cuvres littéraires du Québec.

Suzanne Paradis rend compte du premier recueil de poésie de $S R$ :

Le besoin qu'elle [SR] éprouve de juguler un sentiment dont la puissance l'effraie encore a induit en erreur la plupart de ses critiques. [...] Il semble [...] que Simone Routier ne veuille pas alourdir la poésie des pages sombres de l'amour et de la vie. Son art est tout entier un refuge, une soupape de sûreté, en quelque sorte une porte sur l'exil où le poète cherche beaucoup plus à assurer son équilibre qu'à dénoncer son cœur ${ }^{27}$.

La même critique reprend ce jugement à propos du deuxième recueil de poésie publié par $S R$ :

Le vers est sage, trop strictement ordonné pour animer la blessure dont il voudrait porter les couleurs. Le cri trop bien retenu évite à la fois l'éclat et l'écho. Le poète s'attache bien davantage à cerner des pensées et des réflexions philosophiques qu'à rendre compte de l'état d'un cœur tourmenté par l'amour ${ }^{28}$.

27 "L'Immortel Adolescent" [1928], dans le Dictionnaire des œuvres littéraires du Québec, tome 2: 1900-1939, Montréal, Fides, 1980, p. 583. ${ }^{28}$ "Ceux qui seront aimés" [1931], dans ibid., pp. 195-196. 
À propos du troisième ouvrage de $S R$, un recueil de notules, Suzanne Paradis récidive: «Encore une fois, Simone Routier manifeste le souci du juste milieu qui a marqué son œuvre, l'empêchant peut-être de donner sa mesure profonde ${ }^{29}$ ».

Sous la plume de Lucie Robert, on peut lire ce qui suit au sujet des deux derniers recueils de poésie de $S R$ :

Dans l'un et l'autre recueils, la poésie de Simone Routier s'est arrêtée. $[\ldots]$ Tout ici porte à réaction [...]. L'écriture elle-même refuse d'avancer [...]. Les vers sont classiques, réguliers dans leurs rimes et dans leurs mélodies, témoignant d'un univers où rien ne bouge, où tout est réglé à l'avance, un univers qu'il n'est pas question de remettre en cause. [...] l'impuissance a eu raison de la parole [je souligne $]^{30}$.

Enfin, Suzanne Faguy juge ainsi RDVF: «Il s'agit d'une réplique acerbe, à maints égards méprisante, dans laquelle l'auteur se complaît à dénigrer les réflexions de Thérèse Tardif, du moins à en réduire la portée, apparemment révolutionnaire à l'époque ${ }^{31}$ ».

S'affirme donc de plus en plus cette sécheresse croissante, plus madame Routier vieillit: elle s'est rangée, sagement bien intégrée dans l'honnêteté de la société qui l'entoure et la vénère (ce dont témoignent les nombreuses «reconnaissances» officielles

\footnotetext{
29 "Paris. Amour. Deauville» [1932], dans ibid., p. 828.

30 "Le Long Voyage [1947] et Psaumes du jardin clos" [1947], dans ibid., tome 3: 1940-1959, p. 585.

${ }^{31}$ "Désespoir de vieille fille, pensées de Thérèse Tardif et Réponse a (Désespoir de vieille fille», par Marie de Villiers [sic] (pseudonyme de Simone Routier)", dans ibid., p. 277. - On aurait bien aimé que Suzanne Faguy explicite sa dernière remarque: «[..] la portée, apparemment révolutionnaire à l'époque.» C'est ici que le lecteur attendait précisément un développement nécessaire qui ne vient pas sur cette révolte scandaleuse.
} 
dont elle est la destinataire). Jusqu'à ses vers, de dire la critique, qui sont redevenus bien sages et «classiques».

Comment interpréter pareille évolution? Vieillissant, on se rabat sur les valeurs de son enfance. On revient aux sources consolatrices de l'Origine, de sa famille et de son pays, critères retrouvés de sa conduite. La crise pour ainsi dire «romantique» est passée. On a atteint trente, quarante ans surtout. Il fallait bien devenir rationnel et raisonnable. C'est ainsi par exemple que tous ceux (pas très nombreux d'ailleurs) qui avaient cru à la révolution culturelle, la contreculture des années soixante ${ }^{32}$, ont démissionné, reniant l'idéal que recélait ce mouvement de renouveau radical d'une prise de parole, par exemple dans le nord de l'Ontario. La fatigue doit d'ailleurs y être pour quelque chose..., de guerre lasse comme le veut la vieille expression française. Et la source se tarit, et l'on cesse d'écrire. Mais l'on oublie que sans folie la littérature n'existe plus, l'imaginaire valable ne s'inspirant toujours que du délire comme chez don Quichotte, soit de "l'enthousiasme ${ }^{33}$ " que Platon reconnaissait aux poètes et que précisément pour cette raison il chassait de la cité comme dangereux au bon et sain déroulement des affaires de la république. Certes, tout cela nous semble appréciable dans l'explicitation de la réduction au silence chez madame

\footnotetext{
32 On pensera à la revue, un instant célèbre, de la contre-culture québécoise, Mainmise, ainsi qu'à deux "anciens" pour ainsi dire de Parti pris, Pierre Maheu et Paul Chamberland, dont l'expérience d'une commune fut un échec si pénible.

${ }^{33}$ Se retrouver dans un dieu, être animé, "possédé» par un dieu fou ou ivre, tel Dionysos (vs Apollon). C'est ce silence sur ce délire initial et fondateur qui rend les actuelles interprétations des œuvres si ennuyeuses et futiles, au bénéfice, dit-on, des apparats et des méthodologies critiques.
} 
Routier. Mais, la vraie raison de cette défaite et surtout de la hargneuse fin de non-recevoir qu'elle oppose à $D V F$ de Thérèse Tardif vient en dernière analyse de sa conception de la religion. Deux univers s'affrontent sourdement ici.

Thérèse Tardif pratique une religion expérience, l'expérience de Dieu, alors que Simone Routier s'attache à une religion obéissance, soumission à la volonté de Dieu telle qu'interprétée par son Église. La première consiste en un cheminement personnel vers le Divin, à travers l'humain, tout l'humain ${ }^{34}$. Y persiste une adhésion têtue à l'existence d'un Dieu toujours là, dont on essaie de comprendre des signes de présence en soi et autour de soi. Peut-être pourraiton appeler ce type d'expérience la religion comprise comme trajet, comme aventure mystique. La seconde se veut une conformité à des lois, commandements, directives, venus d'autorités reconnues compétentes dans le domaine de l'éducation de la foi. On dirait du domaine de la Loi dans et selon l'Ancien Testament. Se conformer: le devoir d'état assure la perfection et empêche les déviations malheureuses d'expériences spirituelles aberrantes, tant les subjectivités débridées s'y donnent libre cours. Non contrôlées, pareilles expériences mènent à des pratiques et à des égarements les plus séditieux, dangereux et déplorables: les protestantismes de tout acabit prolifèrent, bientôt tous plus irrationnels les uns que les autres. Ou encore, dix fois pis, naissent, de telles pratiques, des fondamentalismes au bout du compte toujours terroristes! La religion conformiste s'aligne

\footnotetext{
${ }^{34}$ Il faudrait icl répéter, encore et toujours à satiété, le vers inaugural de la tradition «humaniste" en Occident: "Homo sum: humani nihil a me alienum puto" [Je suis homme: rien de ce qui est humain ne m'est étranger] (Térence, Le Bourreau de soi-même, I, 1, 25).
} 
et se modèle sur l'orthodoxie venue depuis Rome. Le bon sens, le sens commun, le «jugement" dans la vie courante, nous l'avons rappelé plus haut, en constituent la pierre angulaire. Un petit esprit quelque peu bourgeois-rationaliste ${ }^{35}$, proposant des évidences irréfutables (l'ancienne apologétique), pénètre ce christianisme du rationnel et du raisonnable; et ce, en dépit du scandale de la croix, folie pour les gentils. L'Église est éminemment sociale et la meilleure gardienne de la santé des sociétés comme des familles, de la moralité publique dont elle seconde et appuie toujours les autorités légales: l'obéissance, aveugle s'il le faut et quand il le faut, devient, selon la si laide expression qu'un style pseudo-littéraire a consacrée, le ciment de l'ordre social si injuste soit-il par ailleurs, et le ciment de l'ordre dans un pays sans lui voué à l'anarchie. Or qui ne le sait, rien de pis que l'anarchie! C'est précisément tout cela, qu'on vient d'esquisser, qui paraît composer le catholicisme de

${ }^{35}$ Il m'aura toujours paru qu'à la lumière, ainsi qu'à la pratique des cours reçus en philosophie et en théologie au Québec dans les décennies quarante et cinquante, et même déjà du temps de la formation du chanoine Lionel Groulx (ce dont témoignent éloquemment ses Mémoires), ait existé le courant que j'évoque icl et qui convoyait à sa manière et bien malgré lui, j'imagine, des données héritées du Siècle des lumières, soit un petit rationalisme, étroit et borné, et mesquin, et des plus prétentieux au point de vue scientifique. Car l'Église, dans ce courant, voulait établir à quel point elle savait parler le langage de son époque et en connaissait bien le savoir. Et cependant l'Église, toujours, pouvait encore, parmi tant de sciences nouvelles, conquérant pour lors leurs titres de noblesse, prouver et prêcher l'existence de Dieu et la valeur des Evangiles, objective et scientifique. Scientifique, décidément ce mot fut le cauchemar de la chrétienté depuis le dix-huitième siècle. Important certes, voire capital, toutefois ce mot (et, évidemment, tout ce qu'il implique et comporte) ne devrait pas obnubiler la pensée chrétienne au point de la figer comme une statue de sel, et ce, pour la bonne raison que science et religion relèvent de deux ordres radicalement incommensurables de connaissance et de pratique, de deux «univers d'être», chacun ayant droit à sa place au soleil de l'Homme, semi-scientifique et semi-mythique, donc mystique. 
madame Routier, la religion perfection (ou conformité), qui laisse bien peu de place à l'évolution lente, par essais et erreurs, d'un cheminement personnel vers Dieu. Cette intelligence de la foi est irréconciliable avec la recherche de $T T$.

Récapitulons. Deux humanismes différents, profondément divergents, deux conceptions de la foi et de la pratique de la religion s'opposent vivement ici, chez deux femmes de la même époque et pour qui, toutes les deux, Dieu demeure la réalité suprême. Pareil contexte de foi vive et vivante, il ne faut jamais l'oublier dans l'interprétation des deux œuvres opposées en nos réflexions. Car ce respect restitue tout simplement la problématique propre et caractéristique, la perspective spécifique où évoluaient la génération tant de la Relève que de Refus global et une certaine fraction, située bien à gauche, de notre élite intellectuelle du Québec autour de la Seconde Guerre mondiale. $S R$ reproche à $T T$ d'abord de manquer de sens commun, de simplicité et de pudeur. L'exhibitionnisme, les plaintes incessantes à propos des amours perdues et de son cour endolori, cette revendication d'avoir droit de s'en prendre à tout et à tous, et dans la même coulée de citer Dieu au tribunal de ses souffrances, ce manque de retenue et de conformité à la volonté de Dieu, toute cette attitude, pareil comportement devant la vie répugne à l'auteur de $R D V F$. Dieu seul ici a des droits et l'homme n'a que la liberté d'obéir: tel est son premier, suprême et ultime devoir. Dans ce cadre, ainsi délimité, $S R$ estime qu'il faut savoir recevoir les coups inévitables que l'existence nous réserve, les supporter stoïquement ${ }^{36}$, mettre toujours «la raison» de son côté et la

${ }^{36}$ Comment ne pas penser ici aux vers de Vigny bien connus en ces 
suivre toujours (on croirait entendre des échos de l'Art poétique de Boileau!), cette raison définie et illuminée par l'Église: l'orthodoxie, définie par Rome, conserve jalousement toute préséance dans la conduite humaine. Ajouter à cela quelques souffrances imposées et recherchées pour expier tout le mal que nous ayons jamais fait et dont l'humanité est responsable $^{37}$.

Ne resterait qu'à prendre et à expliciter quelques textes de RDVF pour appuyer ce qui vient d'être affirmé, et le but modeste de ce petit article serait atteint. Nous nous abstiendrons de compléter ainsi notre analyse. Nous ne ferions que «prouver» ce que nous avons déjà trouvé. Autrement dit, nous tomberions dans la tautologie, bénédiction de nos prétentions! Plutôt, reprenons et approfondissons quelque peu ce que nous avons posé jusqu'ici.

\section{III - Des deuX PRÉSEnCes au Monde}

Selon ma conception toute personnelle et selon ma pratique toute subjective en littérature, la lecture des textes, d'abord sacrés ${ }^{38}$, propose une interprétation

temps-là:

Gémir, pleurer, prier, est également lâche.

Fais énergiquement ta longue et lourde tâche

Dans la vole où le Sort a voulu t'appeler.

Puis après, comme moi, souffre et meurs sans parler.

Alfred de Vigny, "La Mort du loup", Les Destinées, dans Poésies complètes, Paris, Editions de Cluny, «Bibliothèque de Cluny», 6, 1937, p. [166].

${ }^{37}$ Il faut tout de même reconnaître ici que la réflexion s'avère plus que jamais vraie en cette fin du pire siècle que l'histoire de l'homme ait connu sur la terre et de l'horreur duquel il est évidemment (qui d'autre?) le grand auteur.

${ }^{38}$ Sur tout ceci, voir l'ouvrage si éclairant de Werner G. Jeanrond, Introduction d l'herméneutique théologique. Développement et signification, Paris, les Éditions du Cerf, 1995, 270 p.- Le lecteur trouvera là 
qui met en évidence les conditions de possibilité, les postulats d'intelligibilité impliqués dans toute écriture, comme d'ailleurs dans toute conduite ou comportement. Ce sont ces implications sous-jacentes, au fond du texte, et en constituant l'horizon de lecture, que l'analyste cherche à dégager. Ou, en termes beaucoup plus simples, qu'est-ce qui rend un texte intelligible? Par exemple, qu'est-ce qui rend la lecture de Phèdre possible? On répondra que c'est une conception bien particulière du christianisme qui se trouve motrice et inspiratrice, régulatrice, pondératrice de la pièce de Racine. Et cette conception se trouve être la doctrine et, peut-être encore plus déterminant, le climat affectif, générateur du sens, qu'est le jansénisme, vers lequel Racine alors se tournait de plus en plus.

Déterrer ces implications, ou mieux les déduire du texte par un archéologique travail de questionnements de plus en plus serrés et profonds, voir comment des états affectifs, par exemple des mécanismes d'angoisse, d'amour, d'ambition, etc., affectent et construisent subtilement et sournoisement le texte, voilà en propre et trop brièvement le travail de l'herméneutique. Ajoutons que celle-ci peut ne retenir qu'un thème d'étude et d'approfondissement comme, par exemple: quelle conception vécue d'ordre social rend possible tel ou tel roman de Balzac ou de Zola (par contraste)?

Dans les deux œuvres que nous confrontons, le thème fondamental, je le prends comme un postulat de base dont l'évidence s'impose sans plus, c'est bel

ce qu'on entend aujourd'hui par l'herméneutique des textes théologiques ou philosophiques. Ce n'est pas celle-là qui se trouve ici présentée. 
et bien la religion, la «chose religieuse" telle que vécue par les deux auteurs. Maintenant il faut aller plus loin. Qu'est-ce, au fond, vraiment ce qui s'oppose ici? Ce sont deux modes fondamentaux d'êtreau-monde, et d'abord dans le domaine de la connaissance et de la compréhension, sinon de l'intelligence des choses qui entourent l'homme ou qui le constituent de l'intérieur.

En fait, l'influence la plus importante de Jack [Kerouac] sur Gioscia fut de le faire passer - en douceur - d'un mode conceptuel renforcé par une érudition massive à un mode existentiel. Jack ne le fit pas en parlant de la vie, encore une fois, mais en la célébrant ${ }^{39}$.

C'est très exactement cette opposition entre la présence-au-monde conceptuelle et l'expérience compréhensive existentielle qui différencie les deux approches de la religion chez $T T$ et $S R$. Il ne faudrait pas manquer ici de souligner que pareille pratique religieuse, à l'époque de l'engagement de nos deux «héroïnes", totalisait toute leur attitude devant la vie, leur conduite, leur fidélité, leurs convictions, valeurs, affects, etc. La religion s'avérait ici et alors l'ultime et suprême instance de tout le penser et de tout l'agir humains, la catégorie transcendantale des critères directeurs et mobilisateurs de toute une vie et, s'il y a lieu, de sa survie.

Dans un cas, le conceptuel, tout se réduit à l'imitation d'un modèle, comme y invitaient deux directives du Nouveau Testament. Première directive: «N'allez pas croire que je sois venu abolir la Loi [...] ; pas un i, pas un point sur l'i, ne passera de la Loi [...] Celui

${ }^{39}$ Citation tirée de Gerald Nicosia, Memory Babe. Une biographie critique de Jack Kerouac, Montréal, Éditions Québec/Amérique, 1994, p. 617. 
donc qui violera l'un de ces moindres préceptes [...]»; la pratique scrupuleuse du dernier iota de la Loi, assumée plus loin par la charité, mais non pas, jamais évacuée par la charité, cette pratique épuise le comportement sacré. Puis, seconde directive: «Soyez parfaits comme votre Père céleste est parfait» et comme l'enseignait le livre de base de la spiritualité de ces temps de foi: l'Imitation de Jésus-Christ. De plus, remarquons que le modèle d'opération dans le conceptuel est pyramidal et hiérarchique, et commande dès lors une obéissance aveugle à la voix venue d'en haut: ce modèle ne peut souffrir ni démocratisation ni quelque démocratie que ce soit.

Dans le second cas, l'existentiel ${ }^{40}$, la vie divine n'est ainsi inhérente à la vie humaine dans sa quotidienneté qu'en vivant celle-ci intensément, en lui étant fidèle, peu à peu on découvre le divin, l'action du divin, comme le levain dans la pâte; on crée le divin dans sa vie à soi, individu en marche vers l'au-delà de la vie.

C'est quand on en arrive à la pratique «morale» que chacune de ces deux attitudes recèle qu'on verra la vraie différence fondamentale entre elles, ce sera là leur minute de vérité... Et alors, en verra-t-on également l'incompatibilité irréductible. Pour le conceptuel, nous parlerons de Morale, et pour l'existentiel d'Éthique ${ }^{41}$. La morale, rappelons-le, impose une série de codes et de directives, des commandements, auxquels on doit se soumettre pour être parfait. Ces commandements sont d'abord présentés comme venant de la nature elle-même telle que sortie des mains de Dieu au premier moment de

\footnotetext{
${ }^{40}$ Ou mieux peut-être l'existential, auquel je préfère l'expériencial.

${ }^{41}$ Cette dénomination m'est suggérée par le Journal de Charles Du Bos, - encore que lui-même ne l'ait jamais faite ni utilisée telle quelle.
} 
sa création, puis confirmés par la raison et le sens commun, et enfin garantis et assumés par une révélation indiscutable. Le fidèle n'a plus qu'à se conformer, ainsi que nous l'avons montré plus haut. L'attitude imposée par la morale en semble une située à l'extérieur de l'homme et ne paraît pas le rejoindre dans les sources profondes de son être. Elle n'est certainement pas une vie. L'existential, lui, est la vie expérimentée au jour le jour, avec tout le mystère que comporte toujours l'existence. La vérité, ou mieux les vérités, et vérités-pour-soi qu'on y découvre, ne doivent toutefois pas y contredire les préceptes de la morale venus, répétons-le, de la nature-raison-sens commun-révélation. Mais l'existential peut-il connaître pareille limite et orientation venues de l'extérieur du cheminement de l'homme? La connaissance vient ici des situations vécues, de l'expérience. Tout comme c'est le cas dans une expérience d'amour ou d'amitié, de souffrance, voire bientôt de mort. L'intuition fait son chemin par les labyrinthes et l'opacité de la matière traversée ou affrontée $e^{42}$. Le mal, ce qui par la morale est considéré et déclaré mal, ne semble pas dans le second type de connaissance dont il est ici parlé et qui est le propre de la vie tout comme des expériences soumises à nous dans les arts et les lettres, le mal donc, le péché ne semble plus ici mal ou péché. D'où l'éternelle opposition entre, d'une part, les arts et les lettres, l'imaginaire, et, d'autre part, les autres types de savoir et de connaissance.

Il y a deux morales: celle proposée par les lettres et

${ }^{42}$ L'un des meilleurs et des plus beaux exemples de ce phénomène est contenu et expérimenté dans le poème d'Anne Hébert, «le Tombeau des rois», Le Tombeau des rois, présentation par Pierre Emmanuel, Québec, [s.é], 1953, pp. 71-73. 
les arts, ou encore celle de l'imaginaire humain; puis la morale, stricto sensu, celle établie et démontrée par la raison et la révélation. Prenons un exemple, qui ne pourra manquer de soulever bien des oppositions. La masturbation, dans la société catholique qu'était le Québec de 1940 (alors que nos deux auteurs publiaient leurs ouvrages), la masturbation ne fait certainement pas problème dans les arts et lettres, en général, en Occident. Le nu non plus, etc., bref toute la pratique de la chair et du sexe: la "morale» est évolutive et de plus en plus ouverte. Nous avons donc affaire ici à un second et autre système de valeurs, qu'il n'est pas question de développer dans ce court article. Il faut y insister: nous voici donc en présence de deux systèmes de valeurs. Le second, baptisé plus haut Éthique, invite essentiellement à créer et construire sa propre liberté: il est cheminement, assumation lente et patiente et souffrante, humiliante le plus souvent, de nos données les plus profondes ${ }^{43}$. Mais alors, la morale, à l'occasion pour dire le moins, en prendra pour son coup. Le premier système, soit la morale strictement dite, relève du régime de la Loi, et demeure toujours quelque peu inhumaine: l'homme n'est pas fait, ultimement, pour vivre de la Loi et sous la Loi, dans laquelle il s'aliène et qui l'aliène, le rendant faux et artificiel, en même temps qu'elle le rend heureux d'être déchargé du poids de sa libération et de sa liberté dont il n'a plus à assumer la responsabilité. L'homme se trouve alors protégé et sécurisé contre les risques de la liberté, et

\footnotetext{
${ }^{43}$ Jadis, dans son Menaud, maître-draveur (Québec, Librairie Garneau, 1937, p. 67), Mgr Félix-Antoine Savard avait fait interpeller en rêve son jeune Alexis par des paroles sublimes (oubliées aujourd'hui): "délivre la liberté!» - «Où est-elle?» avait demandé le jeune homme. - "Dans ton sang", lui répondirent les grands morts de son pays. "Qu'est-ce qui la retient captive? - Toi-même.»
} 
la femme, «sa» femme, en pareille situation, comme l'ont bien montré l'œuvre et la vie de Claudel, l'encercle et le défend contre le monde et contre luimême, ce que beaucoup d'hommes recherchent dans le mariage.

Il y a donc deux modes d'être-présents-au-monde, deux modes de comprendre, et, plus près ici de nos considérations, deux modes de vivre, soit deux Morales, affirmation énorme, nous le reconnaissons. La seconde, baptisée Éthique tantôt, se développe et se déploie, dévoile les vérités que lui révèle le devenir, à même les expériences humaines les plus profondes dans une vie, à même le mystère d'une existence et, ultimement dans le psychisme humain, à même l'agression, la soif du pouvoir, l'incommensurable vanité humaine, l'instinct sexuel et l'angoissethanatos, soit les grandes instances et pulsions à l'horizon de l'être-homme. Tous les arts et lettres, l'autre moitié de l'âme, moitié dite l'Imaginaire, en témoignent éloquemment.

La deuxième et la troisième parties de la présente recherche semblent sœurs jumelles. Ce qui les différencie tout à fait cependant, c'est l'effort de théorisation que développe la dernière partie. Il y est établi que deux auteurs, vivant dans la même atmosphère religieuse des années quarante, s'opposent radicalement par suite de deux conceptions antithétiques de la religion pratiquée. Ceci prouvé, force est de relever et constater que, de façon sousjacente à leurs attitudes religieuses, les conceptions évoquées réfèrent à une complexité duelle du psychisme humain. Ce qui a la valeur et le mérite de tirer l'audacieuse conclusion qu'existent somme toute deux «Morales», et non deux sources de la morale, et 
non plus la seule interprétation rationaliste et raisonnable du comportement humain, de l'homme qui n'est ni animal ni raisonnable: grâce aux «lettres» on peut fonder une autre morale. Bien plus, voilà qui nous achemine vers la double interprétation des textes littéraires (les seuls auxquels je veuille ici et pour l'instant me limiter).

Au début de son ouvrage, $S R$ «demande à ceux qui aiment Dieu de prier pour vous», TT faut-il l'écrire. Cette belle et très humaine condescendance, si «supérieure" dans sa charité, ne peut que référer à un ordre sûr de lui-même. Mais, sans doute, Dieu n'a-til pas besoin de pareilles supplications pour savoir quoi faire... (du moins dans le cas présent!). Des deux religions, des deux charités, tout comme d'ailleurs des deux interprétations possibles d'un texte littéraire, le lecteur reste tout libre de choisir sa voie, sachant que la première lui vaudra l'approbation de l'orthodoxie, alors que la seconde en fera un hors-laloi, comme $T T$, qui ne sera récupérée que par le sérieux scientifique de l'université, dont les professeurs recevront de gros salaires pour désamorcer le scandale en le constituant, pour le répéter, en objet d'étude objective, donc inoffensif ${ }^{44}$.

\footnotetext{
${ }^{44}$ Tous ceux qui ont si grassement vécu de Rimbaud et de Verlaine, voire de Nelligan et de Saint-Denys Garneau, ou encore de D.H. Lawrence, sur lequel un professeur de littérature anglaise avait rédigé sa thèse de doctorat et quil refusait de mentionner seulement le nom de E. M. Forster parce que celui-ci avait écrit un roman favorable à l'homosexualité... Tirer toute sa dignité sociale et toute l'importance de son statut social, et en vivre généreusement, de ce qu'on peut savamment disserter sur les écrivains maudits de tous les pays, de tous lieux et temps...!
} 
*

**

Addition. Une donnée nouvelle, découverte au moment d'aller sous presse, mérite d'être rapportée ici, car elle lève le voile sur l'accueil que reçut $R D V F$ dans le milieu littéraire d'Ottawa. Dans le journal inédit de Marie-Rose Turcot, écrivain d'Ottawa (18871977), on peut lire en date du dimanche 5 décembre 1943: «Simone [Routier] qui était au thé avait prétexté devoir aller chez les Dominicains pour le cours de philo. Elle est en effervescence par les lettres reçues accusant réception de son livre: Réponse [à Désespoir de vieille fille], dont une de Donatien Frémont qui avait parié un poudrier que l'auteur était Maria [Pouliot] (Charles Maurel). Simone s'amuse de tout, ne se doute pas que son livre est nul et a désappointé tous ses amis qui comptaient la voir revenir avec un livre plus riche en substance poétique.» Ce journal, tenu régulièrement de 1936 à 1972, compte onze cahiers totalisant environ 3500 pages dactylographiées; il est conservé au Centre de recherche en civilisation canadienne-française, Université d'Ottawa. 